\title{
Roles of mTOR-regulating GTPases in regulating drug resistance in senescence-like hepatoma cells
}

Wei Jiang

Xiangya Hospital Central South University

\section{Zhenglin Ou}

Xiangya Hospital Central South University

Qin Zhu

Xiangya Hospital Central South University

Yuanbing Yao

Xiangya Hospital Central South University

Hongyan Zai ( 1029165173@qq.com)

Xiangya Hospital Central South University

\section{Research Article}

Keywords: Senescence, mTOR, GTPase, liver cancer, MEK inhibitor

Posted Date: October 25th, 2021

DOI: https://doi.org/10.21203/rs.3.rs-1014270/v1

License: (c) (1) This work is licensed under a Creative Commons Attribution 4.0 International License. Read Full License 


\section{Abstract}

Radiotherapy and chemotherapy can arrest cancer cells in a senescence-like state, which can lead to therapy resistance and cancer relapse. Despite the cell cycle arrest, senescence-like cells have persistent mTOR activity that is insensitive to nutrient starvation. The mechanisms and functions of mTOR activation in senescence-like cells remains unclear. mTOR is regulated by several small GTPases including the lysosome-localized Rag complex, ER-Golgi-localized Arf1 and Rab1, and endosomelocalized Rab5. In this study, we knocked down these GTPases in both proliferating and senescence-like HepG2 cells induced by X-ray radiation. We then compared mTOR activity and drug resistance to MEK inhibitors. We also examined the roles of autophagy and lysosomal activity in mTOR activation. In addition, by analyzing the Cancer Genome Atlas (TCGA) database, we studied the relationship between the expression levels of these GTPases and the survival of liver hepatoma carcinoma (LIHC) patients. Our results showed that although all GTPases were required for optimal mTOR activation in proliferating HepG2 cells, only Rag is required in senescent-like counterparts. Consistently, the drug resistance of senescent-like cells can be reduced by knocking down of Rag but not other GTPases. Autophagic and lysosomal activity were increased in senescent cells; pharmacological inhibition of autophagy-lysosome decreased mTOR activity and preferentially sensitized the senescence-like HepG2 cells to MEK inhibitors. Therefore, recycling of intracellular materials could be a key mechanism to maintain mTOR activity and promote drug resistance in senescence-like state. In LIHC patients, expression of Rag but not Rab5 or Arf1 was associated with unfavorable prognosis. Our study therefore has defined a key role of Rag GTPase in mediating mTOR activation and drug resistance in senescent-like HepG2 cells, which could have important implications in developing second-line treatments for liver cancer.

\section{Introduction}

Cells become senescent, or aged, after repeated replications. Senescence is traditionally defined as an irreversible cell cycle arrest in G1 phase (G1 exit) triggered by eroded telomeres in aged primary cells [1, 2]. Despite the cell cycle arrest, senescent cells can secrete a variety of bioactive molecules. The functions of these bioactive molecules, termed senescence-associated secretory proteins (SASP), remain poorly studied[3-5]. Senescent cells also showed an elevated $\beta$-galactosidase ( $\beta$-GAL) activity, which has been used frequently to assess cellular senescence. Many other cellular perturbations can also cause senescence or senescence-like phenotype, for example, abnormal chromatin organization, proteostasis, mitochondrial respiration [6-8]. In cancer cells, chemotherapy and radiotherapy can induce oxidative and genotoxic stress, triggering a senescence-like state $[9,10]$. Cancer cells in senescence-like state are known to cause treatment resistance in many types of cancer $[11,12]$. These senescence-like cancer cells, through unknown mechanisms, support tumor cell proliferation, leading to cancer recurrence. Targeting senescence-like cancer cells therefore promises better treatment, but the mechanisms regulating senescence-like state remain unclear.

mTOR has been shown to be involved in senescence establishment [13]. mTOR is a Serine/Threonine protein kinase regulating multiple aspects of cell growth and maintenance, including protein synthesis, 
autophagy, mitochondrial function $[14,15]$. In response to nutrient signals for example, growth hormone, glucose, or amino acid (AA), mTOR is rapidly activated and phosphorylates effector proteins for signal transduction. S6 Kinase is one such effector among others, whose phosphorylation at Threonine-389 (S6K-T389) is frequently used to assay mTOR activity [16]. Persistent mTOR activity is associated with drug resistance in cancer treatments, including MEK inhibitors $[17,18]$. In senescent cells, mTOR was persistently elevated and insensitive to serum and AA starvation $[19,20]$. How mTOR is persistently activated and whether it is related to senescence-induced drug resistance has not been sufficiently investigated.

A serial of small GTPases including Rheb, Rag, Rab1, Rab5, Arf1, GTPase have been shown to regulate mTOR activity in response to nutrients cues. Th most studied one is Rag GTPase. Rag is protein complex, consisting of two sets of heterodimers: Rag A/B and Rag C/D GTPase. It was shown that GTP-bound RagA/B and GDP-bound RagC/D activate mTOR in the absence of AA [21], suggesting the Rag GTPase complex is an important AA sensing switch. Interestingly however, several other studies showed that the Rag GTPases and homologs are not essential for AA sensing, as cells can still sense AA stimulation and activate mTOR in the absence its absence, albeit less efficiently [22-25]. Rab1A GTPase is a well-known regulator of ER to Golgi protein trafficking, which was found to bind and activate mTOR in response to AA [24]. Another ER-Golgi associated GTPase Arf1 and the endosome-associated Rab5 GTPase were also found in a genetic screen to modulate mTOR activation by AA [26], suggesting diverse mechanisms to regulate mTOR under different physiological conditions. Whether and how these GTPase regulate mTOR in senescent cells or senescence-like cancer cells remains unknown.

In this study, we explored the roles of Rab1, Rab5, Arf1, and Rag GTPase in senesce-induced mTOR hyperactivation in HepG2 cells and found that Rab1, Rab5, and Arf1 were not needed for mTOR hyperactivation. Interestingly, the lysosome-associated Rag GTPase remained essential for mTOR activation in senescence-like cancer cells. Knocking down Rag but not other GTPases increased cancer cell killing by MEK inhibitors. Senescence-like cancer cells showed elevated lysosomal activity, inhibition of which rendered senescence-like HepG2 cells sensitive to MEK inhibitors. Elevated expression of RagC but not other GTPases was associated with poor prognosis in liver cancer patient.

\section{Results}

\section{Rag GTPase but not Rab1A, Rab5, Arf1 was required for persistent mTOR activity in senescence-like hepatoma cells}

mTOR was persistently active and insensitive to short-term amino acid (AA) starvation in senescent primary human fibroblasts [20], but and mechanisms remain unclear. We aimed to characterize the roles of small GTPase activators of mTOR including Rag, Rab1A, Rab5, and Arf1 in senescence-like hepatoma cell line HepG2. We first applied $x$-ray radiation to HepG2 cells to obtain senescence-like cells. After 2 weeks post-radiation, cells are arrested and showed significant $\beta$-galactosidase activity, as determined by flow cytometry using Click-iT EdU indicator and CellEvent Senescence Green probe, respectively (Figure 
1A and 1B). Second, we examined mTOR activity in response to AA starvation by Western blotting of S6 kinase phosphorylation (S6K-T389). The results showed that in senescence-like HepG2 cells mTOR activity was much higher than proliferating cells after 15,30, and 60 min of AA starvation (Figure 1C and 1D). Third, we knocked down Rag, Rab1A, Rab5, and Arf1 through specific siRNAs in senescence-like and proliferating HepG2 cells. All GTPase knockdowns reduced mTOR activity in proliferating cells.

Interestingly however, in the senescence-like HepG2 cells, only RagC siRNA significantly decreased S6KT389 levels (Figure 1E-H), suggesting that Rag GTPase was specifically required for mTOR activity in senescence-like state.

\section{Knocking down Rag but not Rab1A, Rab5, Arf1 increased sensitivity of senescence-like hepatoma cells to MEK inhibitors}

Persistent mTOR activity contributes to chemotherapy resistance, including MEK inhibitors. Since our findings showed a specific requirement of Rag but not other GTPases in senescence-like cancer cells, we asked if Rag could be also specifically required for drug resistance. To do this, we treated senescence-like HepG2 cells and proliferating controls with $10 \mathrm{nM}$ trametinib, a well-known MEK inhibitor for 3 hours. We then examined apoptosis and cell death through staining cells with Annexin $\mathrm{V}$ and propidium iodide (PI), respectively, followed by flow cytometry analysis. Consistently with previous studies, senescence-like cells were much resistant than proliferating cells to the MEK inhibitor. With the siRNA treatments, only RagC knockdown significantly increased the sensitivity to the MEK inhibitor in senescence-like HepG2 cells. Long term survival was examined by CellTiter-glo after treating cells with $10 \mathrm{nM}$ trametinib for 3 days. Consistently, only RagC knockdown significantly reduced the survival in senescence-like HepG2 cells. Therefore, we concluded that Rag but not the other GTPases were required for drug resistance phenotype in senescence-like HepG2 cells.

\section{Inhibiting of lysosomal activity reduced mTOR activity and sensitized senescence-like hepatoma cells to MEK inhibitor.}

In response to nutrient limitation, cells activate autophagy to engulf intracellular materials in autophagosome, which is then digested in lysosome to generate free AAs and lipids for cell maintenance. Rag complex is localized to the lysosome and play essential roles in amino acid sensing and mTOR activation. The specific requirement of Rag GTPase in senescent-like cells suggested that hyperactive mTOR and drug resistance could be due to increased lysosomal activity. To test this possibility, we first examined if autophagic and lysosomal activity was increased in senescence-like HepG2 cells. By incubating cells with a lysosome-specific self-quenching substrate (which will emit fluorescence when degraded by lysosomal proteases), our flow cytometry experiments showed that lysosomal activity was robustly increased upon senescence induction (Figure 3A and 3B). Second, we inhibited autophagic and lysosomal activity by hydroxychloroquine (HCQ) and Bafilomycin-A1 (BAF), two well-known autophagylysosome inhibitors. Both drugs successfully reduced lysosomal activity as revealed by flow cytometry and accumulation of autophagic marker LC3 proteins (Figure 3A-D). Indeed, for both inhibitors, 1-hour treatment reduced mTOR activity in senescence-like HepG2 cells but had only slight effect on 
proliferating counterparts (Figure 3E). Consistently, these autophagy-lysosome inhibitors sensitized senescence-like HepG2 cells to MEK inhibitors, but have only slight effect on proliferating HepG2 cells (Figure 3F). Together, these results suggested that senescence-like cancer cells had increased dependency on autophagy-lysosome for their nutrient supply and cellular maintenance.

\section{Elevated lysosomal activity increased Rag-mediated mTOR activation}

Another situation relying on autophagy-lysosome for nutrient supply is starvation. We reasoned if the specific dependency of mTOR on Rag GTPase in senescence-like HepG2 cells was due to autophagic and lysosomal recycling of intracellular components, activating lysosomal activity in proliferating cells should increase the dependency of mTOR on Rag GTPase. To test this, we applied a partial AA starvation strategy, allowing for examination of S6K-T389 that was difficult to detect in full starvation. By decreasing the AAs to $5 \%$ of normal concentration in the medium for 18 hours, we found a significant increase in lysosomal activity in HepG2 cells compared to those cultured in full AA (Figure 4A-B). We knocked down RagC, Rab1A, Rab5, and Arf1 by siRNA and examined S6K-T389 levels by Western blot. All siRNA knockdowns fully inhibited S6K-T389 phosphorylation in normally cultured HepG2 cells (Figure 4CD). Under partial starvation however, only RagC siRNA robustly decreased S6K-T389 levels; other siRNAs showed much reduced effect. Therefore, the results confirmed that cells with elevated autophagic and lysosomal activity increased the dependency on Rag GTPase for mTOR activation, lending further supports to the specific functions of Rag GTPase in promoting mTOR activity and drug resistance in senescence-like HepG2 cancer cells.

\section{RagC was stronger than Rab1A, Rab5 or Arf1 GTPases as a prognostic predictor for unfavorable outcome in LIHC patients}

We investigated into the clinical data regarding the association of the GTPases with liver cancer and overall survival of LIHC patients. RNAseq and survival data from NIH TGCA database were analyzed by using GEPIA, a recently developed web-based bioinformatics tool [27]. By examining the differential expression of RagC, Rab1A, Rab5, and Arf1 in cancer tissues $(n=369)$ and non-cancer controls $(n=160)$, we found that RagC and Arf1 but not Rab1A or Rab5 were significantly elevated in cancer tissues (Figure $4 \mathrm{E})$. The LIHC patients had gone through different treatments including chemotherapy and radiotherapy, which induced senescence-like cancer cells. If our discovery that RagC but not other mTOR regulating GTPases was specifically important for maintaining the survival and drug resistance of the senescencelike HepG2 cells, then higher levels of RagC but not other GTPases should predict poor survival. Indeed, by plotting the Kaplan Meier curve for LIHC patients with the $25 \%$ highest and $25 \%$ lowest RagC expression, we showed that RagC-high patients had significantly worse overall survival (Figure 4F). The hazard ratio was 2.8 , indicating that the RagC-high patients were 2.8 times more likely to die than RagClow patients. By similar standards, higher expression of Rab1A, Rab5, and Arf1 did not show significant $(P<0.01)$ different survival than lower expressing controls. 


\section{Discussion}

Senescent cells are arrested in cell cycle and become resistant to many cancer treatments, including chemotherapy and radiotherapy $[1,2,11,12]$. The underlying mechanisms remain not well understood. mTOR is a key regulator of tumor growth and its persistent activity has been reported to increase chemoresistance and radio-resistance [18]. By comparing the roles of small GTPase regulators of mTOR in proliferating and senescence cells, we show for the first time, in our knowledge, that only Rag, but not Rab1A, Rab5, or Arf1 is required for persistent mTOR activity in senescence-like HepG2 cells. Consistently, Rag but not the other GTPases increases resistance to cancer drugs trametinib, a potent MEK inhibitor currently under clinical trials for several cancer types. We found that Rag GTPase promoted mTOR activity in response to elevated lysosomal activity. Chemicals that inhibit autophagy-lysosome pathway restore the sensitivity of HepG2 cells to MEK inhibitors. Our study therefore has important implications in developing better strategies for liver cancer treatments.

mTOR is the master regulator of cell growth and proliferation. Recently, mTOR has been proposed to mainly senses AA signaling through Rag GTPase [21],. Interestingly, mTOR is localized to multiple subcellular organelles, such as in the Golgi, ER, mitochondria, endosome, and lysosome [28, 29]. mTOR has also been shown to bind directly to chromatins in the nucleus [30, 31]. The broad localization patterns suggest that mTOR could sense nutrients including AA through multiple sites. Consistently, mTOR was regulated by multiple small GTPases including Rheb, Rag, Rab1A, Rab5, and Arf1 [21, 24, 26], further confirming the multiple-site regulation model. Our study in senescence-like cancer cells show that in AA-rich medium all GTPases were required for optimal mTOR activity. However, in senescence-like cells where autophagic and lysosomal activity were increased, Rag GTPase become dominant. The idea is likely that cells sense AA through multiple sites when cultured in rich medium. For example, Rab5 mediates mTOR sensing extracellular AAs trafficked through endosome, Rab1A and Arf1 mediate sensing AA pool for protein synthesis, while Rag GTPase mediates sensing AA broken down by autophagylysosome. When cancer cells enter senescence-like state, mTOR mainly rely on AA supplies from autophagy-lysosome, making Rag the most important mTOR regulators in senescence-like cancer cells. Due to the ever-changing environment, it is hard to imagine that mTOR will solely rely on one source of AAs for cell growth and maintenance. Supporting this, it was shown that Rag GTPase was not essential for mTOR activation [22, 25, 32]. Yeast cells with Rag homolog Gtr1/2 deleted grow normally and remain sensitive to AA stimulation [23,33]. Our study therefore arguing against the current predominant idea that Rag GTPase is only AA sensing mechanism by mTOR [34].

The senescence-like cancer cells have multiple ways to escape treatments. One mechanism is by entering a dormant state where growth and proliferation are mostly halted, causing resistance to drugs that preferentially targeting proliferating cells [35]. In our case, the radiation-induced senescence-like state shows elevated mTOR activity, suggesting that growth signals remain actively functioning. This result is in line with many findings showing that senescent and senescence-like cells, albeit arrested, remain metabolically active [36,37]. Another broadly accepted mechanism of drug resistance is the modulation of tumor microenvironment by releasing SASP. If this contributes to drug resistance to MEK inhibitors in 
our study, one can increase the drug sensitivity simply by removing senescence-like cells. Consistently, RagC knockdown in our study increased apoptotic cell death and at the same time increased drug sensitivity (Figure 2). Other GTPases that did not affect cell death had no effect on drug resistance. Although favor a Rag-mTOR regulation of cell death as a mechanism to reduce the SASPs and drug resistance, we cannot rule out the possibility that mTOR might directly regulate SAPS. Given the pivotal role of mTOR in senescence, future study may investigate if there is a direct connection between mTOR activity and SASP. Our conclusion is derived from experimental results in HepG2 cells; whether this could be applied to other cancer cells remains unknown.

\section{Materials And Methods}

\section{Cell culture and drug treatments}

HepG2 cells were obtained from The Cell Bank of Type Culture Collection of Chinese Academy of Sciences. Cells were maintained on petri dish in Dulbecco's Modified Eagle Medium (DMEM) supplemented with $10 \%$ (vol/vol) fetal bovine serum (FBS), 100 units $/ \mathrm{ml}$ penicillin, and $100 \mu \mathrm{g} / \mathrm{ml}$ streptomycin in a $37^{\circ} \mathrm{C}$ incubator with $5 \% \mathrm{CO} 2$ and moisture. Senescence-like cells were obtained by exposing HepG2 cells to 20 Gy X-ray on petri dish. Fresh medium was added immediately after irradiation then every 3 or 4 days. Senescence was induced after 2 weeks. Starvation was conducted by replacing culture medium with AA and serum free DMEM for 1 hour. For partial AA starvation, cells were changed to AA-free DMEM then supplemented AA Solution (ThermoFisher) to $5 \%$ of suggested concentration for 18 hours. MEK inhibitor trametinib was obtained from Sigma and dissolved in DMSO as $10 \mathrm{mM}$ stock, then diluted in cell culture the final concentration of $10 \mathrm{nM}$ for 3 hours. Lysosome inhibitors Bafilomycin A1 (BAF) and hydroxychloroquine (HCQ) and were added to cells at the final concentration of $100 \mathrm{nM}$ and 100 uM, respectively, for 3 hours.

\section{siRNA knockdown}

siRNAs were purchased from ThermoFisher Scientific. Cells were transfected with siRNA using Lipofectamine 3000 (ThermoFisher Scientific) according to manufacturer's instruction. Briefly, siRNA and lipofectamine 3000 were separately diluted in Opti-MEM medium for $5 \mathrm{~min}$ and mixed. After $20 \mathrm{~min}$ incubation at RT, siRNA and lipofectamine mix were added to cells at $90 \%$ confluency. Medium was changed after 24 hours.

\section{Western blotting}

Cells on petri dish were washed with PBS then lysed with 1X SDS-PAGE sample loading buffer diluted from 4X loading buffer (250 mM Tris-HCl pH 6.8; 8 \% SDS; $0.2 \%$ Bromophenol Blue; $20 \% \beta$ mercaptoethanol; $40 \%$ glycerol). Whole cell lysates were heated at $95^{\circ} \mathrm{C}$ for $5 \mathrm{~min}$ and separated by SDSPAGE and transferred to polyvinylidene fluoride (PVDF) membrane. Western blotting was conducted by blocking the membranes in $5 \%$ non-fat milk then probing with primary antibodies in $5 \%$ non-fat milk for 1 hour. Membranes were washed extensively with PBST (phosphate buffered saline supplemented with 
0.5\% Tween 20), then incubated with HRP-conjugated secondary antibodies for 30 min. After extensive wash with PBST, bound HRP-conjugated secondary antibody was detected by enhanced chemiluminescence (ECL). Antibodies against human mTOR antibody, S6K-T389, and Actin was obtained from Abcam.

\section{Flow cytometry}

Flow cytometry was used to quantify senescence, apoptotic cell death, and lysosomal activity. For senescence, 1E5 cells were washed with ice-cold Flow Buffer (PBS+0.5\% BSA) then fixed in 4\% formaldehyde for $15 \mathrm{~min}$ at room temperature. After permeabilization with $0.5 \%$ Triton X-100 in PBS, cells were incubated with $\beta$-galactosidase substrate CellEvent ${ }^{\mathrm{Tm}}$ Senescence Green and Click-iT Plus EdU for 1 hours in the absence of $\mathrm{CO}_{2}$. Cells were washed in Flow buffer 3 times and analyzed on cytoFLEX $S$ (BECKMAN) using a 488-nm laser and 530-nm/30 filter. Apoptotic cell death was measured by staining cells with Annexin V (indictor for early apoptosis) and propidium iodide ( $\mathrm{Pl}$, indicator for cell death), according to manufacturer's instruction (Sigma). Briefly, cells were collected in to $1.5 \mathrm{~mL}$ Eppendorf tube by trypsin, then blocked in Flow Buffer (PBS supplemented with 1\% BSA) for 1 hours. Cells were then incubated with FITC-conjugated Annexin V for $15 \mathrm{~min}$. Cells were washed with Flow Buffer extensively to remove non-specific binding. PI was added at the final concentration of $1 \mathrm{ug} / \mathrm{ml}$. Lysosomal Intracellular Activity Assay Kit (Abcam) was used to measure lysosomal activity according to product manual. Briefly, cells were incubated with Self-Quenched Substrate for 1 hour in medium supplemented with $0.5 \%$ FBS. Cells were washed in 1X PBS containing and analyzed on flow cytometer (488 nm excitation laser).

\section{TCGA data analysis}

LIHC patient data were originally from TCGA database and were analyzed on the informatics platform GEPIA (www.gepia.cancer-pku.cn). For differential gene expression, $\mid$ Log2FC| $\geq 0.5$ and $P<0.01$ were used as criteria and compared to Match TCGA normal and GTEx data. Data were presented as Boxplots for indicated genes. To compare the prognosis of LIHC patients with high (top 25\%) and low (low 25\%) expression of indicated genes, overall survivals were plotted using Kaplan Meier curve. P values were derived from Log-Rank test.

\section{Statistics}

All experiments were performed multiple times and tested by unpaired, two-tailed Student's t test or twoway ANOVA as indicated. For flow cytometry, cells were gated with the same standard for all sample in each experiment. Data from at least 2 experiments were analyzed with Prism 7 software. For Western blotting, signals were quantified by Image $J$ software and normalized to tubulin control. Data were further normalized to experimental controls for each experiment and fold changes of 3 experiments were analyzed with Prism 7. Significance was defined by $\mathrm{P}<0.01$.

\section{References}


1. Campisi, J. and F. d'Adda di Fagagna, Cellular senescence: when bad things happen to good cells. Nat Rev Mol Cell Biol, 2007. 8(9): p. 729-40.

2. Childs, B.G., et al., Senescent cells: an emerging target for diseases of ageing. Nat Rev Drug Discov, 2017. 16(10): p. 718-735.

3. Birch, J. and J. Gil, Senescence and the SASP: many therapeutic avenues. Genes Dev, 2020. 34(2324): p. 1565-1576.

4. Kumari, R. and P. Jat, Mechanisms of Cellular Senescence: Cell Cycle Arrest and Senescence Associated Secretory Phenotype. Front Cell Dev Biol, 2021. 9: p. 645593.

5. Kuilman, T., et al., The essence of senescence. Genes Dev, 2010. 24(22): p. 2463-79.

6. Pazolli, E., et al., Chromatin remodeling underlies the senescence-associated secretory phenotype of tumor stromal fibroblasts that supports cancer progression. Cancer Res, 2012. 72(9): p. 2251-61.

7. Joy, J., et al., Proteostasis failure and mitochondrial dysfunction leads to aneuploidy-induced senescence. Dev Cell, 2021. 56(14): p. 2043-2058 e7.

8. Moreno, D.F., et al., Proteostasis collapse, a hallmark of aging, hinders the chaperone-Start network and arrests cells in G1. Elife, 2019. 8.

9. Di Micco, R., et al., Oncogene-induced senescence is a DNA damage response triggered by DNA hyper-replication. Nature, 2006. 444(7119): p. 638-42.

10. Collado, M. and M. Serrano, The power and the promise of oncogene-induced senescence markers. Nat Rev Cancer, 2006. 6(6): p. 472-6.

11. Gordon, R.R. and P.S. Nelson, Cellular senescence and cancer chemotherapy resistance. Drug Resist Updat, 2012. 15(1-2): p. 123-31.

12. Thompson, E.L., J.J. Hu, and L.J. Niedernhofer, The Role of Senescent Cells in Acquired Drug Resistance and Secondary Cancer in BRAFi-Treated Melanoma. Cancers (Basel), 2021. 13(9).

13. Xu, S., Y. Cai, and Y. Wei, mTOR Signaling from Cellular Senescence to Organismal Aging. Aging Dis, 2014. 5(4): p. 263-73.

14. Wei, Y., et al., The role of mitochondria in mTOR-regulated longevity. Biol Rev Camb Philos Soc, 2015. 90(1): p. 167-81.

15. Mossmann, D., S. Park, and M.N. Hall, mTOR signalling and cellular metabolism are mutual determinants in cancer. Nat Rev Cancer, 2018. 18(12): p. 744-757.

16. Holz, M.K. and J. Blenis, Identification of S6 kinase 1 as a novel mammalian target of rapamycin (mTOR)-phosphorylating kinase. J Biol Chem, 2005. 280(28): p. 26089-93.

17. Wang, B., et al., Targeting mTOR signaling overcomes acquired resistance to combined BRAF and MEK inhibition in BRAF-mutant melanoma. Oncogene, 2021. 40(37): p. 5590-5599.

18. Murugan, A.K., mTOR: Role in cancer, metastasis and drug resistance. Semin Cancer Biol, 2019. 59: p. 92-111.

19. Zhang, H., et al., Mitogen-independent phosphorylation of S6K1 and decreased ribosomal S6 phosphorylation in senescent human fibroblasts. Exp Cell Res, 2000. 259(1): p. 284-92. 
20. Carroll, B., et al., Persistent mTORC1 signaling in cell senescence results from defects in amino acid and growth factor sensing. J Cell Biol, 2017. 216(7): p. 1949-1957.

21. Sancak, Y., et al., Ragulator-Rag complex targets mTORC1 to the lysosomal surface and is necessary for its activation by amino acids. Cell, 2010. 141(2): p. 290-303.

22. Efeyan, A., et al., RagA, but not RagB, is essential for embryonic development and adult mice. Dev Cell, 2014. 29(3): p. 321-9.

23. Stracka, D., et al., Nitrogen source activates TOR (target of rapamycin) complex 1 via glutamine and independently of Gtr/Rag proteins. J Biol Chem, 2014. 289(36): p. 25010-20.

24. Thomas, J.D., et al., Rab1A Is an mTORC1 Activator and a Colorectal Oncogene. Cancer Cell, 2016. 30(1): p. 181-182.

25. Jewell, J.L., et al., Metabolism. Differential regulation of mTORC1 by leucine and glutamine. Science, 2015. 347(6218): p. 194-8.

26. Li, L., et al., Regulation of mTORC1 by the Rab and Arf GTPases. J Biol Chem, 2010. 285(26): p. 19705-9.

27. Tang, Z., et al., GEPIA: a web server for cancer and normal gene expression profiling and interactive analyses. Nucleic Acids Res, 2017. 45(W1): p. W98-W102.

28. Carroll, B., Spatial regulation of mTORC1 signalling: Beyond the Rag GTPases. Semin Cell Dev Biol, 2020. 107: p. 103-111.

29. Betz, C. and M.N. Hall, Where is mTOR and what is it doing there? J Cell Biol, 2013. 203(4): p. 56374.

30. Kantidakis, T., et al., mTOR associates with TFIIIC, is found at tRNA and 5S rRNA genes, and targets their repressor Maf1. Proc Natl Acad Sci U S A, 2010. 107(26): p. 11823-8.

31. Wei, Y., C.K. Tsang, and X.F. Zheng, Mechanisms of regulation of RNA polymerase III-dependent transcription by TORC1. EMBO J, 2009. 28(15): p. 2220-30.

32. Kim, Y.C., et al., Rag GTPases are cardioprotective by regulating lysosomal function. Nat Commun, 2014. 5: p. 4241.

33. Thomas, J.D., et al., Rab1A is an mTORC1 activator and a colorectal oncogene. Cancer Cell, 2014. 26(5): p. 754-69.

34. Sabatini, D.M., Twenty-five years of mTOR: Uncovering the link from nutrients to growth. Proc Natl Acad Sci U S A, 2017. 114(45): p. 11818-11825.

35. Recasens, A. and L. Munoz, Targeting Cancer Cell Dormancy. Trends Pharmacol Sci, 2019. 40(2): p. $128-141$.

36. Frasca, D., et al., Effects of cellular senescence on metabolic pathways in non-immune and immune cells. Mech Ageing Dev, 2021. 194: p. 111428.

37. Wiley, C.D. and J. Campisi, From Ancient Pathways to Aging Cells-Connecting Metabolism and Cellular Senescence. Cell Metab, 2016. 23(6): p. 1013-1021. 
A

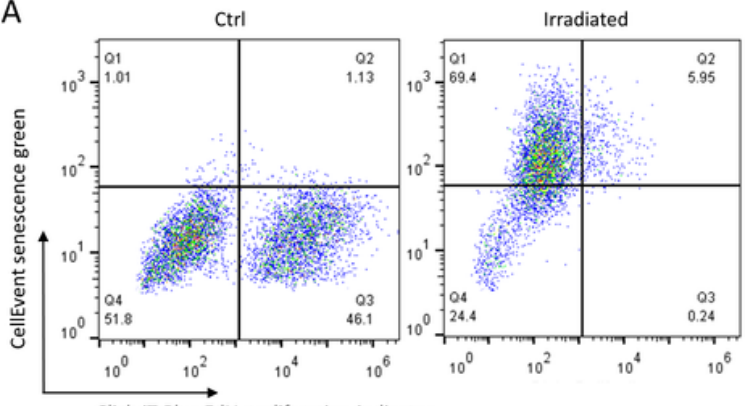

Click-iT Plus EdU proliferation indicator

D

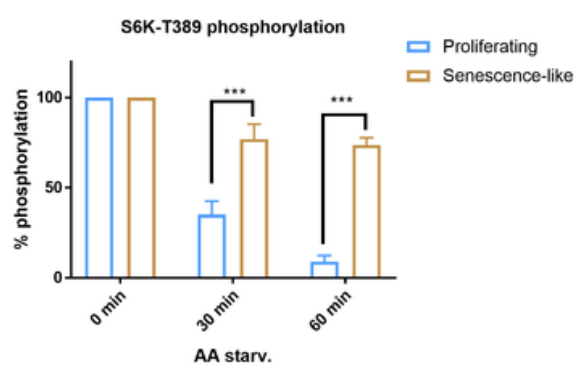

G

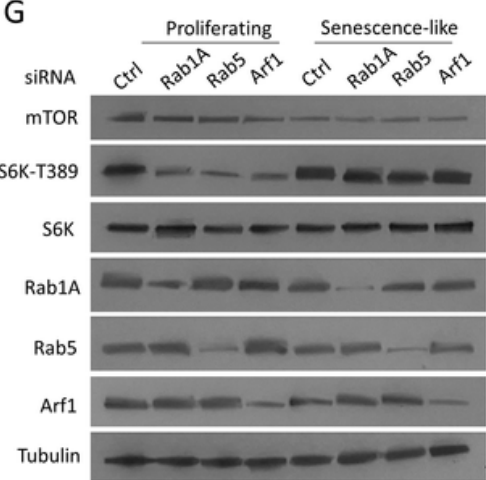

$\mathrm{H}$
B

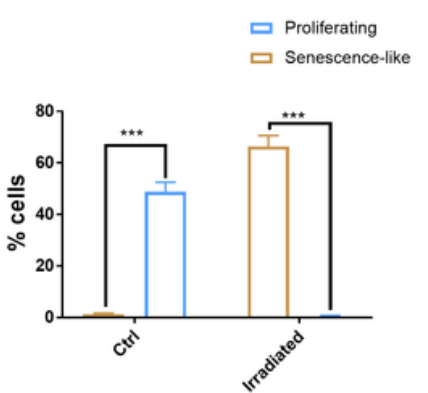

C

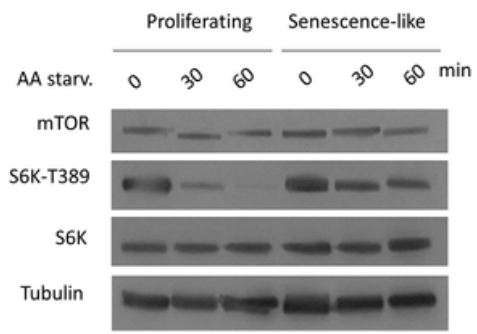

$\mathrm{F}$

$\mathrm{E}$

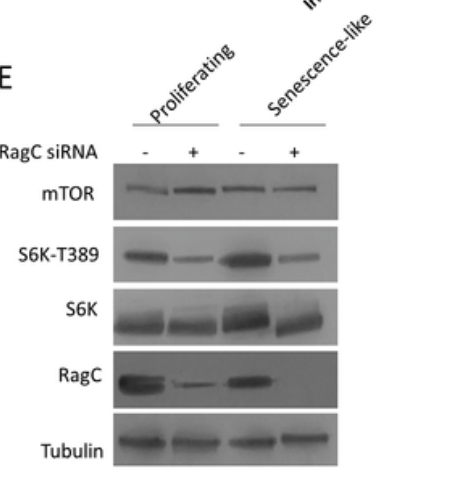

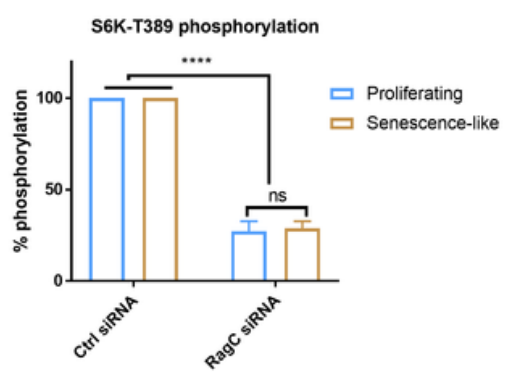

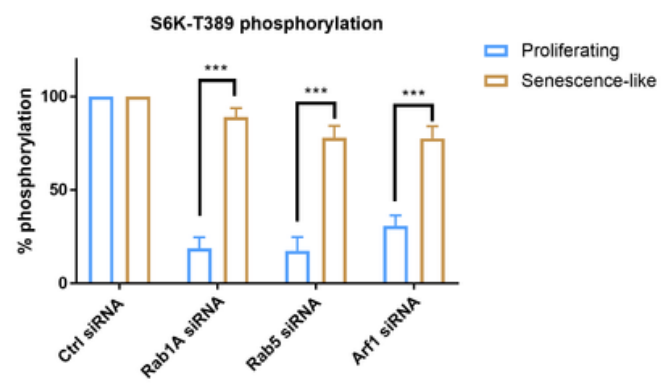

\section{Figure 1}

Rag GTPase but not Rab1A, Rab5, Arf1 was required for persistent mTOR activity in senescence-like hepatoma cells (A) HepG2 cells were irradiated with 20 Gy X-ray and maintained on fresh medium over 2 weeks. Non-irradiated cells and irradiated cells were examined for senescence marker (CellEvent senescence green) and proliferating marker (Click-iT Plus EdU) by using flow cytometry. All samples were gated through the same standard. Representative data are shown. (B) Quantification of senescence-like cell populations from 2 separate experiments. $P<0.001(* \star \star)$. (C) mTOR activity was not sensitive to AA starvation in senescence-like HepG2 cells. Proliferating and senescence-like HepG2 cells were cultured in medium without AA and serum for 30 min or $1 \mathrm{hr}$. Total proteins were separated by denatured SDS-PAGE. S6K phosphorylation at T389 was examined by Western blotting. Representative data are shown. (D) Quantification of S6K-T389 in experiments shown in C (N=3). $\mathrm{P}<0.001(* \star *)$. (E) Persistent mTOR activity was dependent on Rag GTPase. RagC was knocked down by siRNA for 48 hours and S6K-T389 was 
examined by Western blotting. Representative data are shown. (F) Quantification of multiple experimental results in $E(N=3)$. Student's $t$ test $P<0.0001{ }^{(\star \star * *)}$, ns, not significant. $(G)$ mTOR activity was not sensitive to knockdowns of Rab1A, Rab5 and Arf1 expression in senescence-like HepG2 cells. Gene expression were knocked down by transfect cells with specific siRNAs for $48 \mathrm{hrs}$ and S6K-T389 and indicated proteins were examined by Western blotting. Representative data are shown. (H) Quantification of S6KT389 phosphorylation in multiple experiments $(N=3)$. Student's t test $P<0.001(* \star *)$.

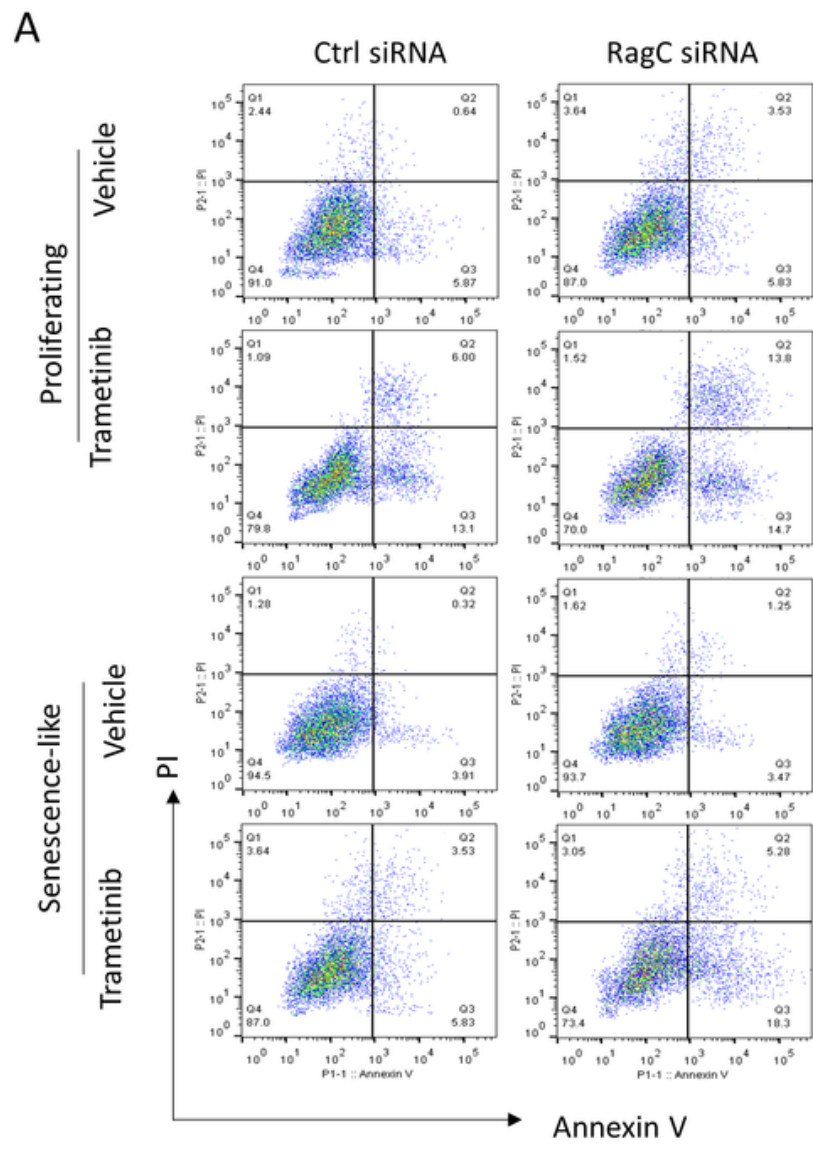

B

$$
\begin{aligned}
& \text { Proliferating }\left[\begin{array}{l}
\square \text { Vehicle control } \\
\text { Trametinib }
\end{array}\right. \\
& \text { Senescence-like }[\square \text { Vehicle control }
\end{aligned}
$$

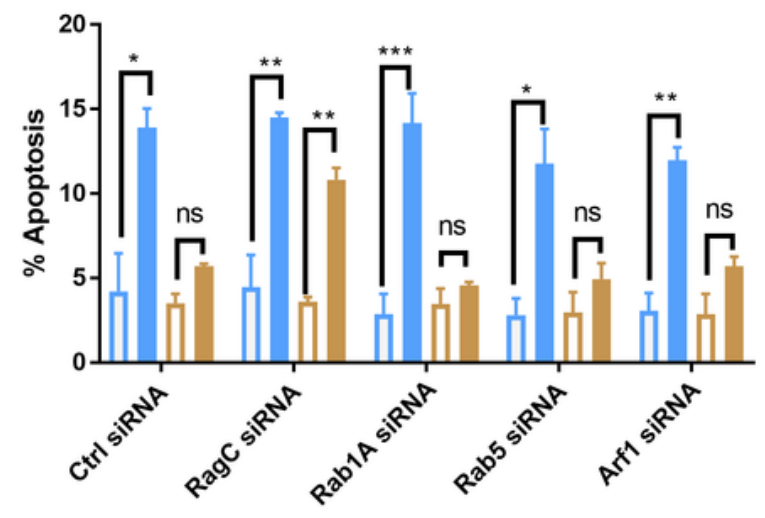

C
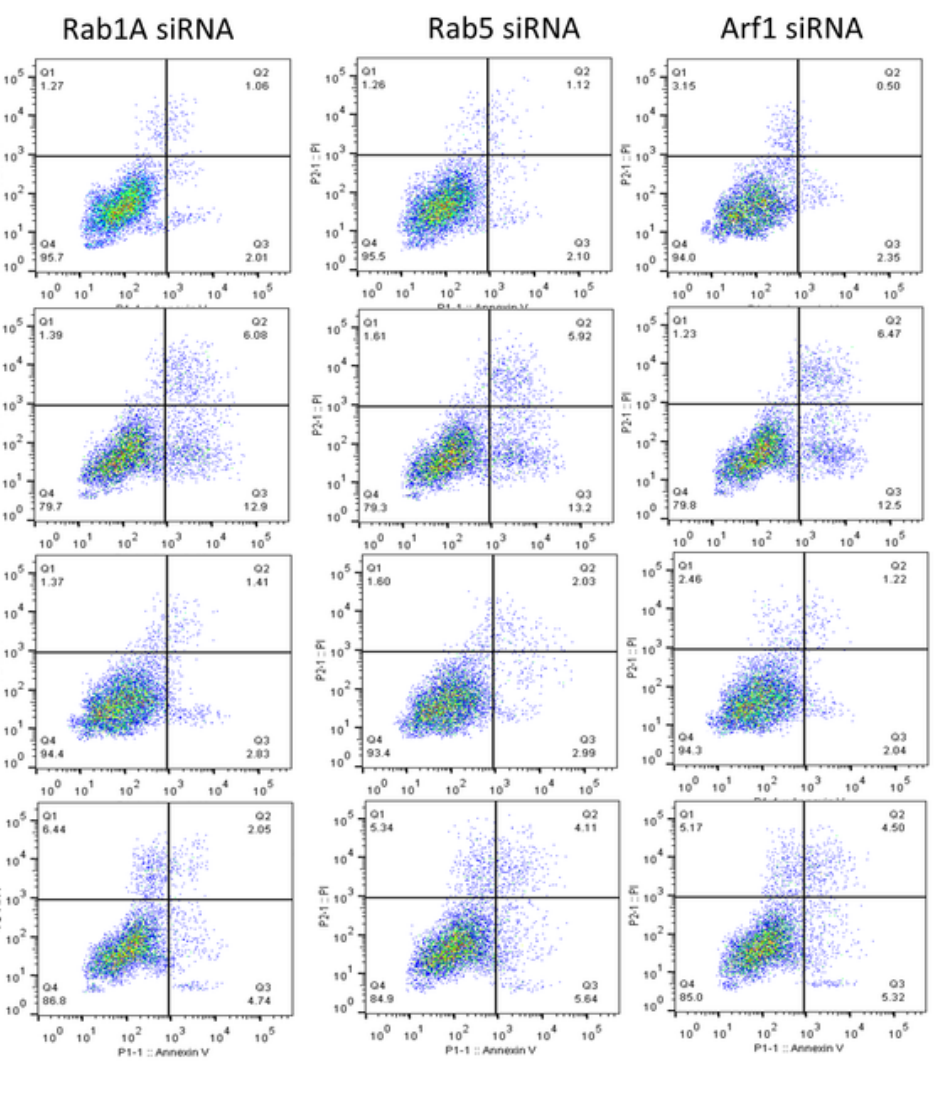

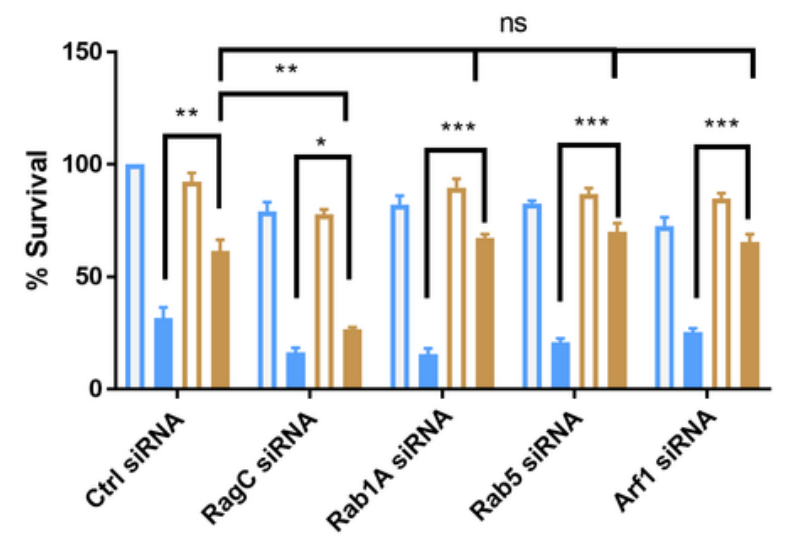

Figure 2 
Knocking down Rag but not Rab1A, Rab5, Arf1 increased sensitivity of senescence-like hepatoma cells to MEK inhibitor. (A) Indicated gene expression were knocked down by siRNA in proliferating and senescence-like HepG2 cells, then treated with $10 \mathrm{nM}$ Trametinib for $3 \mathrm{hrs}$. Apoptosis and cell death were examined by staining cells with Annexin- $\mathrm{V}$ and propidium iodide (PI) followed by flow cytometry.

Representative data are shown. (B) \% apoptosis was quantified for 2 experiments and statistically analyzed by Student $t$ test. $P<0.05\left({ }^{*}\right), 0.01(* \star)$, not significant (ns). (C) Viability was determined by CellTiter-glo after 3 days Trametinib $(10 \mathrm{nM})$. Data from multiple experiments $(\mathrm{n}=3)$ were quantified and analyzed by student $t$ test. $\left.P<0.05(*), 0.01(\star \star), 0.001{ }^{(\star \star *}\right)$, not significant (ns).

A
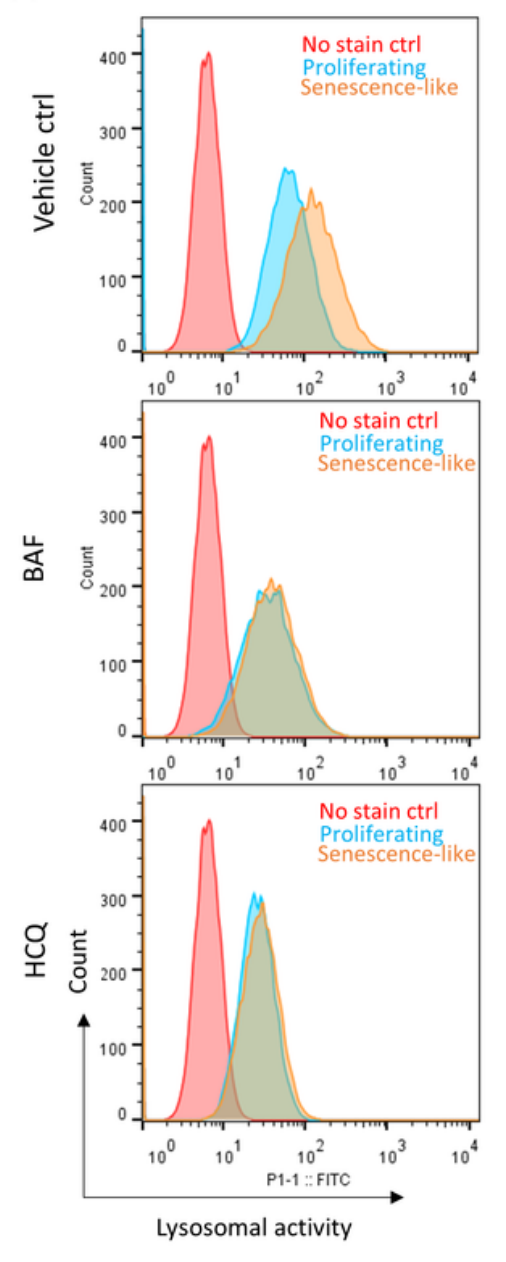

B

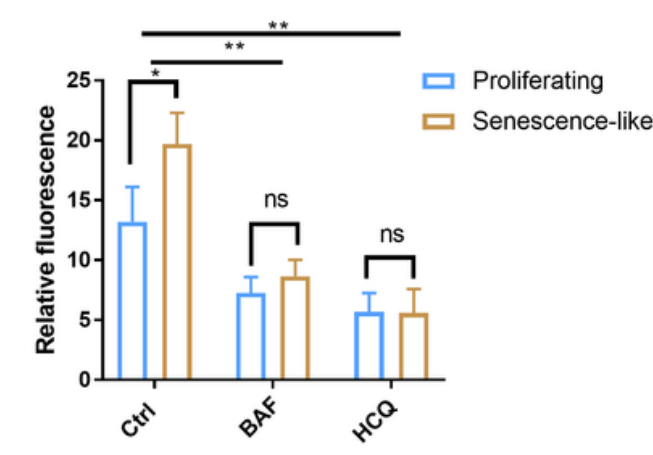

D

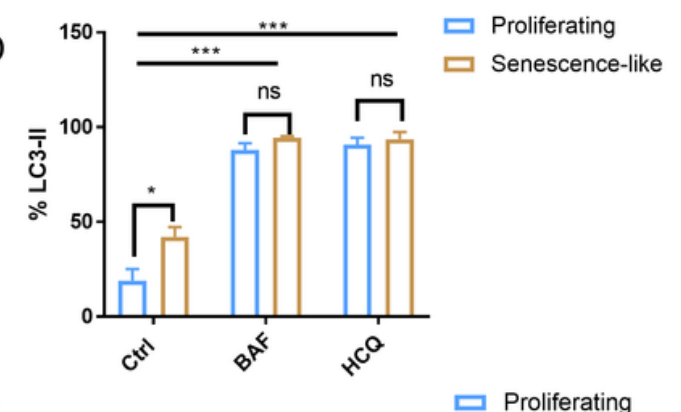

E

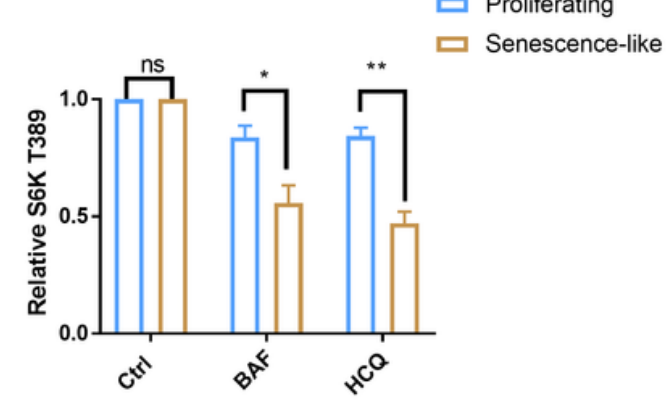

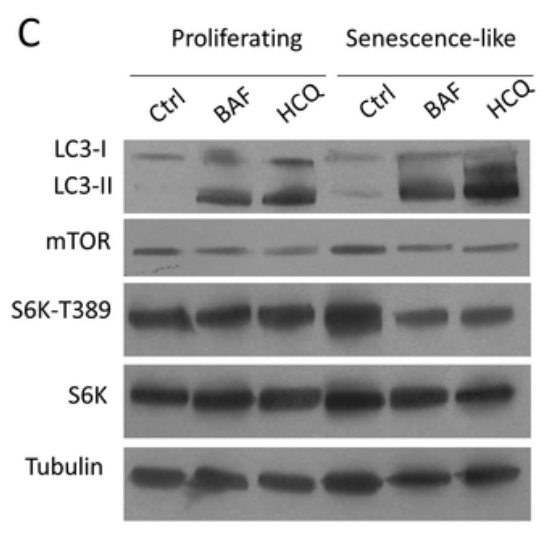

$\mathrm{F}$ Proliferating $[\square$ Vehicle control Senescence-like $\left[\begin{array}{ll}\square \text { Vehicle control } \\ \text { Trametinib }\end{array}\right.$

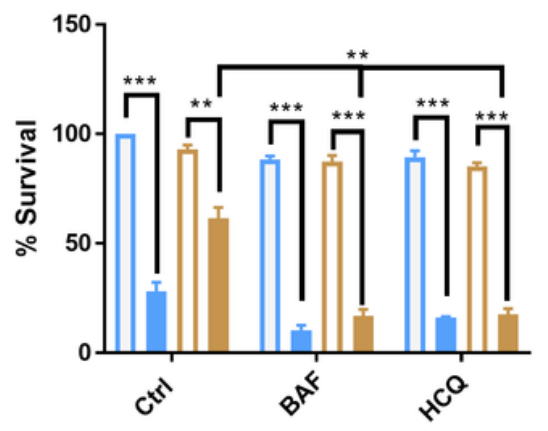

\section{Figure 3}

Inhibiting lysosomal activity reduced mTOR activity and sensitized senescence-like hepatoma cells to MEK inhibitor. (A) Flow cytometry confirmed the inhibition of lysosomal activity by bafilomycin A1 (BAF) and hydroxychloroquine (HCQ). Cells were treated with $100 \mathrm{nM} \mathrm{BAF}$ and $100 \mathrm{uM} \mathrm{HCQ}$ for 3 hours and stained with a self-quenched fluorescent substrate in Lysosomal Intracellular Activity Assay Kit (Abcam). Senescence-like HepG2 cells showed elevated lysosomal activity. No stained controls were used as reference. Shown are representative data. (B) Quantification of multiple experimental data shown in A. 
Student $t$ test. $P<0.05(*), 0.01(* \star), 0.001(* \star *)$, not significant $(\mathrm{ns})$. (C) Inhibiting lysosomal activity reduced mTOR activity in senescence-like but not proliferating HepG2 cells. Cells were treated with $100 \mathrm{nM}$ BAF and $100 \mathrm{uM} \mathrm{HCQ}$ for 3 hours and indicated proteins were examined by Western blotting. Accumulation of LC3-II indicated the lack of lysosomal degradation. Shown are representative data. (D) Quantification of LC3-II accumulation in $\mathrm{N}=3$ experiments. Accumulation of LC3-II indicated the lack of lysosomal degradation. Student $t$ test. $P<0.05\left(^{(}\right), 0.001(* * *)$, not significant (ns). (E) Quantification of S6K-T389 phosphorylation, an indicator of mTOR activity. $\mathrm{N}=3, \mathrm{P}<0.05\left(^{(}\right), 0.01(* *)$, not significant (ns). (F) BAF and HCQ treatment sensitize senescence-like HepG2 cells to MEK inhibitor Trametinib. Cells were treated with $10 \mathrm{nM}$ Trametinib and $100 \mathrm{nM}$ BAF or $100 \mathrm{uM} \mathrm{HCQ}$ for 3 days and survival were examined by CellTiterglo. Data from $N=3$ experiments were quantified. Student's $t$ test $P<0.01(* *), 0.001(* \star *)$. 
A

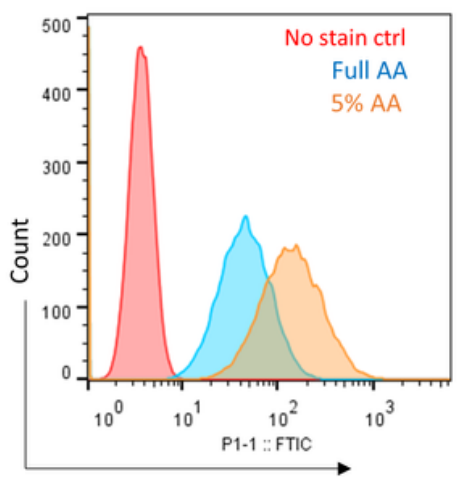

Lysosomal activity

D

$$
\begin{aligned}
& \text { ש Rab1A siRNA } \\
& \text { — RagC siRnA 口 Rab5 siRnA }
\end{aligned}
$$

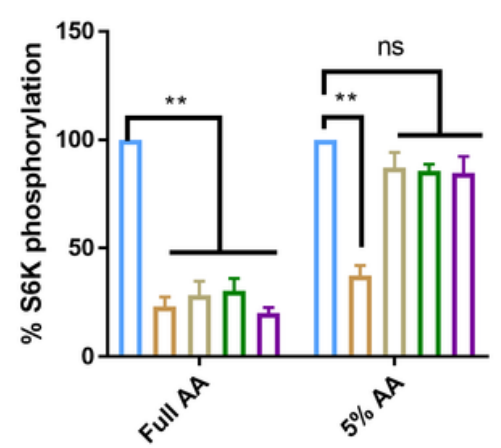

B

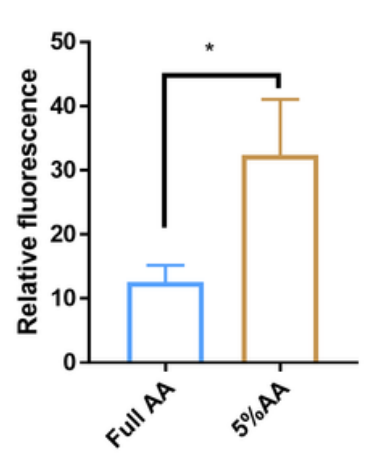

C

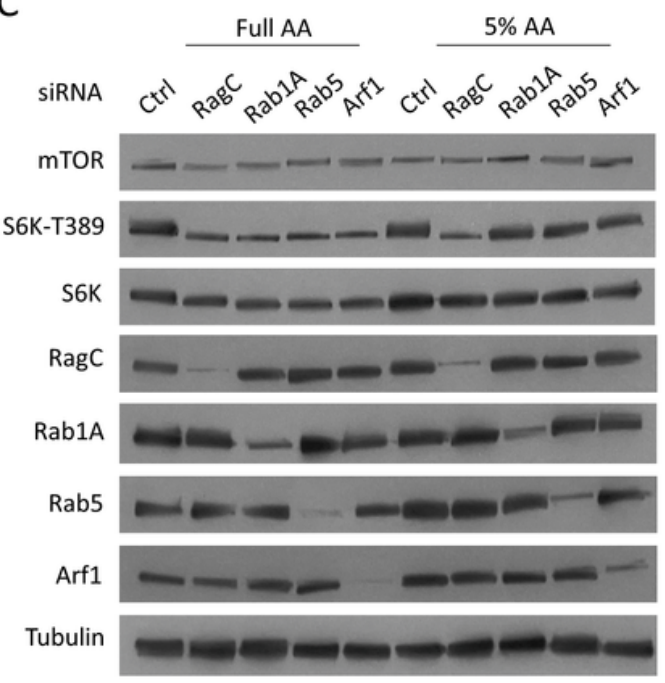

E

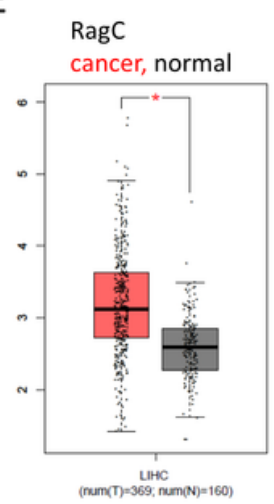

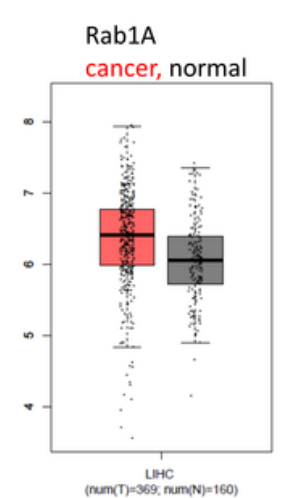

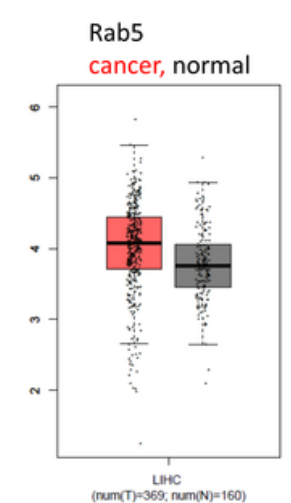

Arf1

cancer, normal

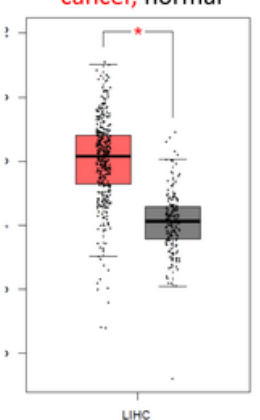

$\underset{\text { UnUm(T)e369: num(N)=160) }}{\text { (n) }}$
F

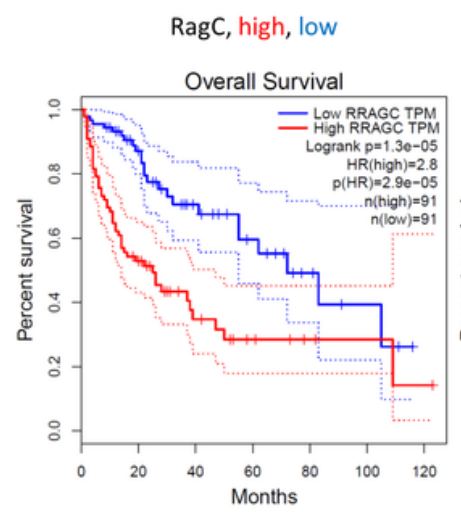

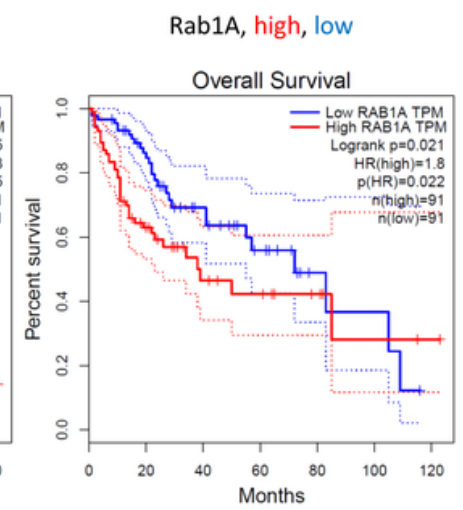
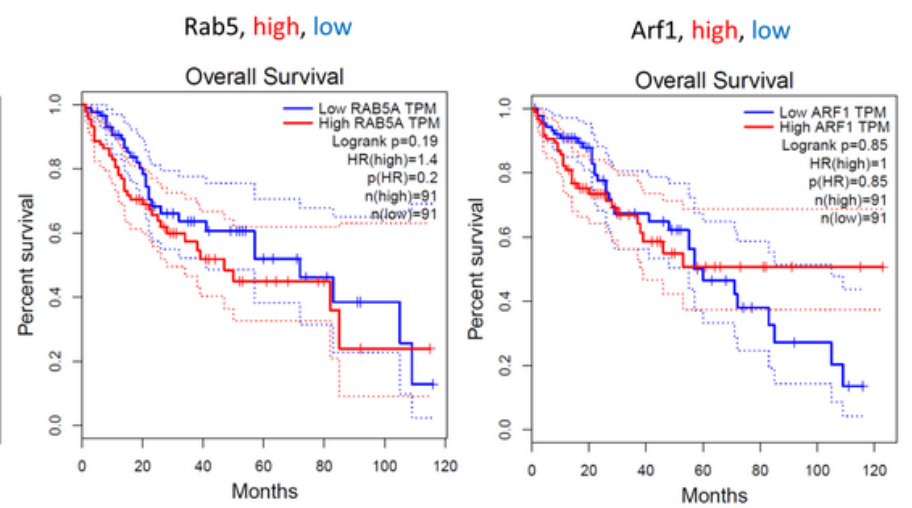

\section{Figure 4}

Rag activated mTOR in response to elevated lysosomal activity and predicted worse survival in LIHC patients. (A) AA starvation increased lysosomal activity. HepG2 cells were starved in culture medium with only $5 \%$ of normal AA for 18 hours, stained with lysosomal activity probe and analyzed by flow cytometry.

(B) Quantification of $N=2$ experiments in A. Student's t test $P<0.05(*)$. (C) mTOR was sensitive to RagC but not Rab1A, Rab5 and Arf1 knockdown in HepG2 cells with elevated lysosomal activity (induced by 5\% AA starvation). Indicated proteins were examined by Western blotting. Representative data were shown.

(D) Quantification of data from $\mathrm{N}=3$ experiments in C. $\mathrm{P}<0.05\left(^{*}\right), 0.01(* *)$, not significant (ns). (E) 
Expression of indicated genes in $\mathrm{N}=369 \mathrm{HepG} 2$ cancer patients and $\mathrm{N}=160$ matched normal controls. $\left.\mathrm{P}<0.01{ }^{*}\right)$. LIHC patient data from TGCA were analyzed with GEPIA bioinformatics tools. (F) RagC but not other GTPases is a robust prognostic predictor for LIHC patients. Overall Survival (OS) data of LIHC patients with top $25 \%$ high ( $\mathrm{N}=91)$ and $25 \%$ low ( $\mathrm{N}=91)$ expression of indicated gene were plotted in Kaplan Meier curves and tested by Log-rank test. $\mathrm{P}<0.01$ was considered significant. HR, hazard ratio. TPM, transcript per million. 